\title{
Aproximación a las discapacidades específicas de aprendizaje en la competencia matemática en el contexto educativo colombiano
}

\author{
Approximation to specific learning disabilities in mathematical competence in the \\ colombian educational context
}

\section{Aproximação as deficiências específicas na aprendizagem da habilidade matemática no contexto educativo colombiano}

\author{
Rafael Enrique Rojas-Ferreira ${ }^{1}$
}

Forma de citar: R.E. Rojas-Ferreira, "Aproximación a las Discapacidades Específicas de Aprendizaje en la competencia Matemática en el contexto educativo colombiano", Eco.Mat. vol. 7, no. 1, pp. 121-140, 2016.

Recibido:

Mayo 13 de 2015

Aceptado:

Septiembre 1 de 2015

${ }^{1}$ Magister en Educación con énfasis en Gerencia Educativa Rafaelrojas1973@msn.com Coordinador educativo del Instituto Técnico Mercedes Abrego Cúcuta-Colombia

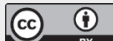

\section{Resumen}

El artículo tiene como objetivo ofrecer una revisión crítica de la literatura sobre el estado de la cuestión relativa a las discapacidades de aprendizaje en Matemática en el contexto educativo colombiano. Para ello, se realizó un recorrido histórico breve de los constructos “discapacidad", “competencia matemática" y "discapacidad de aprendizaje”. Se planteó un análisis de los conceptos, causas, características, evaluación e intervención relacionadas con las discapacidades específicas de aprendizaje en la competencia Matemática. Los diferentes informes del sistema educativo en Colombia, las pruebas nacionales e internacionales reflejan un alarmante porcentaje de estudiantes con discapacidades de aprendizaje en el conocimiento Matemático y evidencian la necesidad de establecer una detección temprana, ya que esta se origina a partir de la primera infancia en el proceso de enseñanza-aprendizaje y se mantienen a lo largo del proceso educativo sino hay intervención oportuna por parte del profesorado, mediante un diagnóstico, seguimiento y evaluación permanente con criterios que orienten la corrección de las dificultades mediante aprendizajes que desarrollen la creatividad, las competencias, la organización y adaptación de contenidos junto a la evaluación autentica y los resultados de aprendizaje como herramientas pedagógicas, entre otras, que permiten establecer a los docentes las medidas curriculares y organizativas necesarias para atender las necesidades educativas que presenta el alumnado con discapacidad especifica de aprendizaje en matemáticas, acordes al nivel y ritmos de su aprendizaje. Se enfatiza la necesidad del reconocimiento, concreción y desarrollo de la categoría de discapacidad de aprendizaje a nivel legislativo y en las políticas educativas públicas en Colombia para una atención de calidad por parte de las administraciones educativas y otros entes, mediante una sólida fundamentación teórico y empírico proporcionada a nivel científico, y con activa participación por parte de los educadores como investigadores en el aula.

Palabras clave: Competencia matemática, Discapacidad, discapacidad de aprendizaje, discapacidad específica de aprendizaje en matemática, trastorno específico del aprendizaje. 
No. 1

Enero-Diciembre 2016 ISSN 1794-8231 E-ISSN $2462-879$ PP: $121-140$

\begin{abstract}
The article aims to provide a critical review of the literature on the state of the art about learning disabilities in Mathematics in the Colombian educational context. To do this, a brief historical review of the constructs "disability", "mathematical competence" and "learning disability" were carried out. An analysis of the concepts, causes, characteristics, evaluation and intervention related to the specific learning disabilities in the Mathematics competence was proposed. The different reports of the educational system in Colombia, national and international tests reflect an alarming percentage of students with learning disabilities in Mathematical knowledge and evidence the need to establish an early detection, since this originates from early childhood in the teaching-learning process and are maintained throughout the educational process if no prompt intervention is provided by teachers, through a diagnosis, monitoring and permanent evaluation, using criteria in order to correct the difficulties through learning that develop creativity, skills, the organization and adaptation of contents along with authentic assessment and learning results as pedagogical tools, among others, allowing to take curricular actions needed to attend the educational necessities of students with DEAM, according to the level and rhythms of student learning. The necessity for the recognition, concretion and development of the category of learning disability at the legislative level and in the public education policies in Colombia for a quality care by educational administrations and other entities is emphasized, through a solid theoretical and empirical foundation provided at a scientific level, and with active participation of educators as researchers in the classroom.
\end{abstract}

Keywords: Disability, mathematical competence, learning disability, specific learning disability in math, specific learning disorder

\section{Resumo}

O artigo tem como objetivo fornecer uma revisão crítica da literatura sobre o estado da questão da deficiência na aprendizagem da matemática no contexto educacional colombiano. Para isso, foi realizada uma breve história do construções "deficiência", "competência matemática" e "dificuldade de aprendizagem". uma análise dos conceitos, causas, características, avaliação e intervenção relacionada com dificuldades de aprendizagem específicas em proficiência de matemática foi levantada. Os vários relatórios do sistema educacional na Colômbia, eventos nacionais e internacionais refletem uma porcentagem alarmante de alunos com dificuldades de aprendizagem no conhecimento matemático e demonstrar a necessidade de detecção precoce, já que este se origina desde a infância o processo de ensino e aprendizagem e são mantidas durante todo o processo educacional, mas nenhuma intervenção atempada por parte dos professores, pelo diagnóstico, monitoramento e critérios de avaliação em curso para orientar a correção de dificuldades, aprendendo a desenvolver a criatividade, habilidades, a organização eo conteúdo adaptação com autênticos resultados de avaliação e aprendizagem como ferramentas educacionais, entre outros, que permitem que os professores a estabelecer medidas curriculares e organizacionais para atender às necessidades educacionais apresentados por estudantes com DEAMs, compatível com o nível eo ritmo de sua aprendizagem. a necessidade de reconhecimento, realização e desenvolvimento da categoria de dificuldade de aprendizagem a nível legislativo e nas políticas públicas de educação na Colômbia para cuidados de qualidade por parte das autoridades de educação e outros organismos, é enfatizada por uma fundação teórica e empírica sólida forneceu um nível científico, e de participação activa educadores e pesquisadores na sala de aula.

Palavras-chave:, a competência matemática, deficiência, dificuldade de aprendizagem, inabilidade de aprendizagem específica em matemática, dificuldades de aprendizagem específicas. 


\section{Introducción}

En esta época se puede aseverar que los conocimientos, el razonamiento y las habilidades de la Matemática son tan importantes como las de lectoescritura. Además, la competencia Matemática es tan esencial en un espectro extenso de disciplinas, profesiones y ámbitos de la vida que ha logrado impactar el vertiginoso avance científico y tecnológico en los últimos dos siglos de la humanidad. Sin embargo, es oportuno mencionar de acuerdo a [1], citados por Blanco [2], que los desarrollos en la investigación educativa en la Matemática son menos intensos que en lectura, tal vez por la complejidad de la tarea y al amplio número de dominios que pueden influir en la práctica de dicha competencia.

Esa complejidad de la investigación en Matemática permite una gran cantidad de interrogantes en el contexto educativo colombiano y permitirán orientar este ensayo. Se intentará responder a inquietudes tales como: ¿Por qué para unos estudiantes es más fácil aprender Matemática que otros estudiantes? ¿Incide el lenguaje en el aprendizaje de la Matemática?, ¿Por qué hay tanta repitencia, deserción y fracaso escolar en esta competencia?, ¿A que responden los bajos resultados de las pruebas estandarizadas Saber y Pisa en Colombia en este conocimiento?, ¿Es verdad que muchas personas nacieron sin tener la competencia en dicha área?, ¿Qué es una Discapacidad Específica de Aprendizaje en Matemática (en adelante DEAM)?, y ¿Qué hace el estado de Colombia para paliar esas Discapacidades de Aprendizaje (DA) en el sistema educativo y en otros sectores?

De lo anterior se puede decir que muchas investigaciones apuntan a explicar lo que está pasando con la Matemática y sus DA desde diversas percepciones negativas en la sociedad, que van en primer lugar, del desinterés, confusión, rechazo y prejuicio para aprender porque no les gusta o no pueden comprender los conceptos elementales de la Matemática; en segundo lugar, a docentes que mal preparados en las universidades imparten enseñanzas que perjudican el aprendizaje y el interés de tales conocimientos en el alumnado; en tercer lugar, a un currículo $\mathrm{y}$ un sistema educativo obsoletos y poco aterrizados en la realidad y los intereses de los ciudadanos; y por último, la visibilidad de las personas con discapacidad (PcD) que carecen de los fundamentos más primordiales en Matemática para adquirir y ejercitarse en conceptos, métodos, estrategias y técnicas de aprendizaje que se aprenden en los diferentes grados escolares y de formación profesionales, $\mathrm{y}$ que, a pesar de tener muchas veces, una inteligencia media o superior al promedio, padecen una DEAM en la formación de las competencias académicas tan indispensables como el conteo, el cálculo y la resolución de problemas.

Luego, la importancia de las DA es clave como lo manifiesta Siegel [3], citado por[2], porque si no se identifica que estudiantes no están en condiciones de seguir los aprendizajes y contenidos curriculares al igual ritmo de sus compañeros en un aula regular, entonces se puede estar exigiéndoles más de lo que pueden dar y se puede llegar a tener instituciones educativas inhumanas que incapacitan las habilidades, talentos y destrezas de los estudiantes porque aprenden más lento, y como afirma Blanco se "podrían calificar erróneamente a algunos, y provocando que éstos puedan acomodarse al estatus de «especiales» y no se esfuercen para superar su retraso" [2]. ISSN 1794-8231 -ISSN 2462 - 8794 PP: $121-140$

Este hecho plantea que los estudios acerca de las DA y las DEAM sean un tema relevante y de gran interés al existir diferentes posturas teóricas y metodológicas en relación a la problemática para determinar claramente criterios unificadores para la definición, explicación, identificación, 
evaluación, diagnostico e intervención de las mismas, y unido a esto, a una carencia de una normatividad integral e incluyente de dicha condición discapacitante que ofrezca respuestas educativas integrales y efectivas para mejorar estos déficits y sobre todo para entenderlos y prevenirlos.

Por tal motivo, tanto las DA y las DEAM se han convertido en médula de preocupaciones, reflexiones e investigaciones que adelanta la comunidad de profesionales de diversos campos como la medicina, la neurología, la psicología, la psiquiatría, la política, y de la educación, entre otras áreas. Estos investigadores están alarmados a causa de la presencia permanente y crecimiento paulatino de estadísticas de personas que la padecen; a los bajos resultados constantes en pruebas internacionales y nacionales; a un elevado número de repitencia, deserción y fracaso escolar; y a la cada vez más acentuadas deficiencias de aprendizaje que se presentan en las instituciones educativas durante los años de formación en la competencia Matemática, que están ocasionando graves impedimentos para la vida cotidiana como al contexto escolar para los proyectos personales y profesionales a lo largo de la vida, en especial, para una PcD.

\section{Metodología}

Este documento se apoya en la investigación documental, concebida por la Upel como: "el estudio de los problemas con el propósito de ampliar y profundizar el conocimiento de su naturaleza, con apoyo, principalmente, en trabajos previos, información $\mathrm{y}$ datos divulgados por medios impresos, audiovisuales o electrónicos [4]. Y es un artículo de revisión de tema que, según el Índice Bibliográfico Nacional Publindex de Colciencias en sus criterios generales de clasificación, se considera es producto de la revisión crítica de la literatura sobre un tópico determinado e intenta establecer qué se sabe del tema, qué se ha estudiado y qué perspectivas permanecen ocultas.

Para la realización de este artículo de revisión sobre las DA y las DEAM en una primera etapa, se propuso buscar, seleccionar, y consultar aquellos recursos que fueran de utilidad para la elaboración de posibles respuestas a las inquietudes planteadas, según la óptica del autor, teniendo en cuenta publicaciones primarias (libros, trabajos científicos en revistas, investigaciones de estudios de caso, artículos de revisión, legislación educativa del tema, intercambios con especialistas, entre otros), y secundarias, relacionadas con las definiciones, explicaciones y desarrollos sobre los constructos investigados. Se efectuó una búsqueda automatizada en bases de datos como Redalyc, Scielo, Dialnet, Scopus, google académico, entre otras. Se utilizaron como descriptores: Discapacidad, discapacidad de aprendizaje, matemática, competencia matemática, discapacidad especifica de aprendizaje, discapacidad especifica de aprendizaje en matemáticas, dificultades de aprendizaje, trastornos de aprendizaje, legislación educativa en discapacidad, políticas públicas en discapacidad, educación inclusiva, accesibilidad, normativas en salud para discapacitados, Colombia y la discapacidad. En una segunda etapa, se compiló y extrajo la información más relevante del material elegido, es decir, aquel elemento teórico relacionado con la revisión descriptiva de la literatura que nos permitió llegar a un análisis y reflexión de la importancia del conocimiento, explicación y del manejo de los conceptos e interrogantes propuestos. Como resultado se realizó una investigación con un enfoque cualitativo y una revisión critica del estado del conocimiento, empleándose un criterio constructivista en que el factor de la intersubjetividad, le ofreció al autor entender la realidad social dando como respuesta el análisis, la sistematización e integración de resultados sobre el objeto de estudio. 


\subsection{Aproximación a las Discapacidades Específicas de Aprendizaje en la competencia Matemática}

\subsubsection{La discapacidad, legislación y sistema educativo colombiano}

Se parte de reconocer que en el proceso de enseñanza-aprendizaje, la competencia Matemática es sin duda una de las habilidades académicas más esenciales y complejas para el desarrollo integral del ser humano, dado el carácter instrumental de esta. Esto lo confirma Muñoz, Novo y Espiñeira [5], quienes concluyeron que la formación integral de un ser humano es el proceso central de la educación. Y en el caso del sistema educativo, las políticas y prácticas del Ministerio de Educación Nacional (MEN) que se han implementado en los últimos años han permitido cerrar las brechas de las dificultades en el aprendizaje de los estudiantes a nivel general. Y esto es un logro importante para Colombia, puesto que responde a uno de los objetivos de desarrollo sostenible de la Organización de las Naciones Unidas (ONU) en los cuales se ha avanzado positivamente.

En tal sentido, parafraseando a Andreas Schleicher, director de Educación y Habilidades para la OCDE y coordinador de PISA, se subraya que los estudiantes que no reciben los fundamentos básicos en matemática, están siendo abandonados y condenados a la marginalización al negársele la posibilidad de ser ciudadanos competentes porque tendrán menos oportunidades de participar productivamente en el trabajo al no estar equipados con las herramientas que necesitan para ser competitivos en una bolsa laboral con permanente evolución.

Aunado a lo anterior, se recuerda que, en el siglo XXI, un caso de dicha marginalización esta dado en el tema de la discapacidad, que en Colombia ha logrado permear todos los escenarios de la sociedad en los últimos sesenta años, fundamentalmente por el aumento de la misma y los factores sociales, ambientales, económicos, culturales y políticos que afectan a la población estudiantil en situación de discapacidad en su entorno familiar y escolar.

La Organización Mundial de la Salud (OMS) [6] señala que, a nivel global, la cantidad de personas con algún tipo de discapacidad es mayor a mil millones, en otras palabras, un $15 \%$ de la población del planeta; y es una cantidad que va en aumento al estar ligada con las enfermedades crónicas y el envejecimiento.

En Colombia para el año 2012, la población total de personas que presentaron alguna condición de discapacidad se encontraba alrededor de 2.149.710, correspondiente al $4,7 \%$ de la población según el DANE [7] y sitúa a Colombia como el segundo país con mayor crecimiento del fenómeno en América Latina, después de Brasil.

Dimensionar lo que es la discapacidad en Colombia como en el mundo, es una tarea difícil por sus múltiples aristas e implicaciones en lo económico, educativo y político, tal vez siendo Shakespeare [8], quien mejor expresa este dilema cuando afirma que:

\footnotetext{
... la discapacidad es un fenómeno multidimensional complejo y escalar. La exclusión social y la devaluación de las personas con discapacidad es un fenómeno mundial, generalizado y persistente. El desarrollo de la fe más incuestionable de los beneficios es la identificación adecuada del fenómeno de la discapacidad, la mejora en la participación y la calidad de vida de las personas con discapacidad. [9]
}

Schriner [9], citado por Gómez Acosta [10], reconoce que: "algunas características de la discapacidad, como ser de naturaleza social, estar determinada por fuerzas demográficas y culturales, enfatizan la imposibilidad de encontrar una definición que pueda dar cuenta del fenómeno de la misma forma en todos los tiempos y lugares". Y es la Convención Internacional sobre los Derechos de las Personas con Discapacidad de las Naciones Unidas de 2008 quien más se acerca a una definición de discapacidad al mencionar que:
Enero-Diciembre 2016 ISSN 1794-8231 E-ISSN 2462 - 8794 PP: $121-140$ 
"surge de la interacción de las características de las personas con las barreras que la sociedad pone a sus diferencias" [11]. Dicha Convención, destacó algunas definiciones interesantes para el movimiento de derechos humanos de las PcD. En el literal e) del Preámbulo de la Convención reconoce que:

[...] la discapacidad es un concepto que evoluciona y que resulta de la interacción entre las personas con deficiencias y las barreras debidas a la actitud y al entorno que evitan su participación plena y efectiva en la sociedad, en igualdad de condiciones con las demás [11].

De igual manera, [11] menciona que la discapacidad puede ser permanente, temporaria o episódica. Se puede afectar a las personas desde el nacimiento o puede ser adquirido más tarde a través de algún accidente o enfermedad. Por lo tanto, cualquier persona que tenga algún déficit que perjudica la participación activa y segura en una comunidad es considerada una PcD.

Para [10], la definición sui generis vigente en la arena de las discusiones teóricas en el ámbito de la discapacidad, es la determinada por la naturaleza relacional que elaboró la Clasificación Internacional del Funcionamiento, la Discapacidad y la Salud (CIF) y/o el Instituto de Medicina de los Estados Unidos (IOM). El modelo IOM para Brandt y Pope [12] identifican la discapacidad como el producto de la interacción entre la persona y el ambiente En este orden de ideas, el modelo biopsicosocial ${ }^{1}$ de la discapacidad se define según [10], como: "el nivel de movimiento y desplazamiento que pueden tener los factores contextuales o el ambiente en la tarea de soportar las características de los individuos". Por esto, la gradación de la

${ }^{1}$ Según la CIF, el modelo "biopsicosocial", define la discapacidad, desde el punto de vista relacional, como el resultado de interacciones complejas entre las limitaciones funcionales (físicas, intelectuales o mentales) de la persona y del ambiente social y físico que representan las circunstancias en las que vive esa persona. ... incluye deficiencias, limitaciones en la actividad y restricciones en la participación, denotando los aspectos negativos de la interacción entre un individuo (con una condición de salud) y la de los factores contextuales individuales (factores ambientales y personales). discapacidad depende de la relación que vive una persona con el ambiente.

Entendiéndose la necesidad de determinar para Colombia de una definición propia de discapacidad que no es un problema únicamente de significado porque de acuerdo a Mitra, [13], citado por [10] que: "alterar la definición teórica de discapacidad puede traer implicaciones sociales, económicas y políticas"

Lamentablemente a partir de estas implicaciones, la discapacidad en el plano conceptual y práctico ha desarrollado múltiples lecturas en razón a tiempos y espacios definidos en Colombia, aunque se mantiene para [10] el modelo individual o médico, que continúa abordando la problemática de la discapacidad desde la deficiencia, asumida como una condición médica para referirse a la privación o deficiencia de un miembro, organismo o mecanismo del cuerpo. A este respecto la CIF apunta a considerar a la discapacidad como: "un problema de la persona, directamente causado por una enfermedad, trauma o condición de salud, que requiere cuidados médicos prestados en forma de tratamiento individual por profesionales" [14]. Advierten Cuervo y Trujillo [15] que dichos tratamientos realmente marcan y deciden la existencia de los sujetos que las sufren.

En Colombia, según [10] es posible ubicar las tendencias frente a la definición de discapacidad en las políticas vigentes y en otras manifestaciones relacionadas. Llama la atención que el estado colombiano está intentando en estos últimos treinta años mediante la reestructuración de su sistema normativo y educativo en todos sus sectores consolidar políticas públicas para las personas en situación de discapacidad. Y en la Constitución Política de Colombia de 1991, expresa Moreno [16], que es donde mejor se puede comprobar la existencia de una normatividad instrumentalizada en por lo 
menos 65 desarrollos jurídicos que pretenden garantizar la inclusión social y la vida digna de las PcD.

Siguiendo este auge jurídico, es el Ministerio de Protección Social en 1993, quien decretó con la ley 100 de Seguridad Social en Colombia, que las personas en situación de discapacidad se definen como: "personas con limitación que con base en certificación médica autorizada no puedan gozar de un empleo competitivo que le produzca ingresos al menos equivalentes al salario mínimo legal vigente", y entre otras normas decreto la Resolución 3165 de 1996, que adopta los lineamientos de atención en salud para las personas con deficiencias, discapacidades y minusvalías.

Así mismo, la Ley General de Educación de 1994, identificó a personas con limitaciones físicas, sensoriales y psíquicas, como parte de los grupos poblacionales de modalidad educativa. Para ello, los artículos 46, 47 y 48 abordan aspectos referidos a integración educativa, apoyo $\mathrm{y}$ fomento $\mathrm{y}$ aulas especializadas, respectivamente, relacionados con la población en situación de discapacidad, indicando las leyes que regulan el servicio educativo estatal para lograr el cumplimiento de una función social conforme a las necesidades y los intereses de las personas, de la familia y de la sociedad.

Por otro lado, al observarse detenidamente la Constitución Política Nacional de 1991, que se fundamenta en el Estado Social de Derecho, Garay [17] demuestra que existen graves limitaciones de diferente orden que descartan a grupos sociales de las oportunidades de desarrollo, ejercicio de la ciudadanía y acceso a bienes y servicios. Esas limitaciones constitucionales trataron de ser resueltas con dos documentos legislativos que marcan la inclusión social de las PcD en la segunda década del siglo XXI, estos son:
- El Conpes Social No. 166, 2013, Política Pública Nacional de Discapacidad e Inclusión Social, el cual contiene los lineamientos de política y el financiamiento de las acciones a cargo de las diferentes entidades del orden nacional involucradas, y que se encuentra vigente desde el presente año, hasta el año 2022. Adopta el enfoque de derechos y el modelo social de discapacidad, que agrupa las barreras de la discapacidad en la sociedad y no en el individuo. Esta política estatal comprende la discapacidad ya no como algo propio de la persona, sino como un complejo conjunto de condiciones creadas por el contexto. Por tal razón, propone que las actuaciones se deben dar bajo un enfoque social, y está orientada a modificar el entorno para garantizar la participación de las PcD en la vida social.

- Ley Estatutaria 1618 del 2013, por medio de la cual se instauran las disposiciones para avalar el pleno ejercicio de los derechos de las personas con discapacidad. Establece que el Registro para la Localización y Caracterización de las Personas con Discapacidad (RLCPD), es la única fuente de datos oficial y válida para identificar a las personas con discapacidad en Colombia, a cargo del Ministerio de Salud y Protección Social, cuya función principal es recolectar información continua y actualizada de las personas con discapacidad, para localizarlas y caracterizarlas en los departamentos, distritos, municipios y localidades del país.

Cabe destacar como lo señalan Martínez, Uribe y Velázquez [19] que con la Ley Estatutaria 1618 del 2013², el Congreso de Colombia, brindo un concepto actual de "personas con y/o en situación de discapacidad", las cuales define como:

[...] aquellas personas que tengan deficiencias físicas, mentales, intelectuales o sensoriales a mediano y largo plazo que, al interactuar con diversas barreras incluyendo las actitudinales, puedan impedir su participación plena y efectiva en la sociedad, en igualdad de condiciones con lo demás [19]

El origen de la Ley es dado por la necesidad de prestar mayor atención al cumplimiento de los derechos y disminuir el índice de toda clase de discriminación a las PcD. Dicha norma es la que actualmente protege en todos los ámbitos, los derechos de las PcD. Aunque se aprecia que la Ley Estatutaria 1618 se queda corta en el sector educativo al no tocar las discapacidades de aprendizajes, y al globalizar los diversos tipos de discapacidades en un único concepto paraguas de las Necesidades Educativas Especiales (NEE), con el propósito de salvaguardar en todos los ámbitos los derechos de las PcD, pero sin profundizar ni desarrollar claramente en ella,

${ }^{2}$ Ley Estatutaria 1618 de 2013, por medio de la cual se establecen las disposiciones para garantizar el pleno ejercicio de los derechos de las personas con discapacidad. Congreso de Colombia. Ministerio de Salud y Protección Social. Bogotá: Ministerio de Salud y Protección Social.
Enero-Diciembre 2016 ISSN 1794-8231

E-ISSN $2462-8794$ PP: $121-140$

\section{7


No. 1

\section{Aproximación a las discapacidades específicas de aprendizaje en la competencia matemática en el \\ contexto educativo colombiano}

las responsabilidades de los diferentes órdenes y organismos nacionales, departamentales y regionales.

Por ende, la legislación en Colombia es excesiva como se ha mencionado en este recuento jurídico y esto advierte que los planificadores y formuladores de las políticas públicas pareciera que le falta mayor contacto e información relacionada con la realidad de las PcD. Ese enmarañado jurídico de políticas impide que el destino de los recursos y acciones del estado no sean eficaces para alcanzar el bienestar de esta población, ya que se traslada a las instituciones y sectores que brindan servicios a las PcD a no prestar o a no efectuar un buen manejo de sus servicios [20], [21] y mantener en una discriminación negativa $^{3}$ especialmente, a una gran cantidad de estudiantes con discapacidades de aprendizaje, que siguen sin tenerse en cuenta en la normatividad actual.

En resumen, el estado de Colombia pretende con los desarrollos jurídicos y con políticas relacionadas con el sistema educativo en general y en especial con las NEE, a mejorar el acceso y participación de todos los colombianos a una educación sin ningún tipo de discriminación y exclusión al darle forma al marco jurídico de la educación inclusiva, que establece el cumplimiento del derecho a educarse con calidad en instituciones educativas abiertas e incluyentes a las poblaciones diversas y en situación de vulnerabilidad aunque siguen presentándose dificultades y vacíos en el momento de la atención a dicha población.

\footnotetext{
${ }^{3}$ Entiéndase en este caso, aquella discriminación que la ley etiqueta a las PcD en NEE, que al tratarlos a todos igualmente sin tener en cuenta su identidad, necesidades, problemas y atenciones de diversa índole, son determinados con unas mismas características "relevantes o especiales" que no lo son para ellos, por ejemplo el caso de los estudiantes con discapacidades de aprendizaje, por ende, se les está condenando a perpetuar su problemática al no ser diferenciados en el sistema educativo y de salud por su situación de discapacidad especifica.
}

\subsubsection{La competencia de Matemática}

Dichos cambios y mejoras de las políticas públicas en el sector educativo se han concretado con una serie de documentos que han trasformado la educación colombiana. Inicialmente son documentos como los Logros e indicadores de logros, y los lineamientos curriculares, con los cuales el MEN en 1998, orientó la formulación del currículo e introdujo tres aspectos para su organización: procesos generales, conocimientos básicos y contextos. Luego amplio a los Estándares Básicos de Competencias publicados por el MEN en 2006, que se sustenta en los lineamientos curriculares y propone estándares de competencias para conjuntos de grados. Y junto a lo mencionado antes, el Instituto Colombiano para el fomento de la Educación Superior (ICFES) y el MEN han contribuido al marco curricular, concretamente con los lineamientos de las pruebas Saber por el ICFES y la matriz de referencia. Últimamente salieron a la luz, los derechos básicos de aprendizaje del MEN, los cuales han sido elaborados y puestos a la comunidad educativa en el año 2015, y que se convierten en herramientas que ofrecen a las familias, colegios y educadores de Colombia, la oportunidad de conocer lo que el futuro ciudadano colombiano debe saber en general desde primero hasta 11 grado. Todo este desarrollo documental tiene como eje articulatorio, el concepto de competencia, como se presenta en la Figura 1.

Destaca Bruno D'amore et al. [22] que la aplicación del concepto "competencia" se ha instalado agresivamente en el discurso de la educación matemática, y con mayor énfasis en el esfera del desarrollo curricular, de la práctica de la enseñanza y la evaluación, donde es frecuente el uso de la expresión de "enseñar por competencias". En este contexto para [22], competencia viene a ser: "la capacidad de afrontar un complejo, o de resolver una actividad compleja", y 
que es posible de ser evaluada. Y es de esta manera, que las competencias están asociadas a la capacidad de afrontar problemas en actividades significativas y complejas por parte del estudiante.
Enero-Diciembre 2016 ISSN 1794-8231 E-ISSN 2462 - 8794 PP: $121-140$

\section{Competencias como eje articulador República de Colombia}

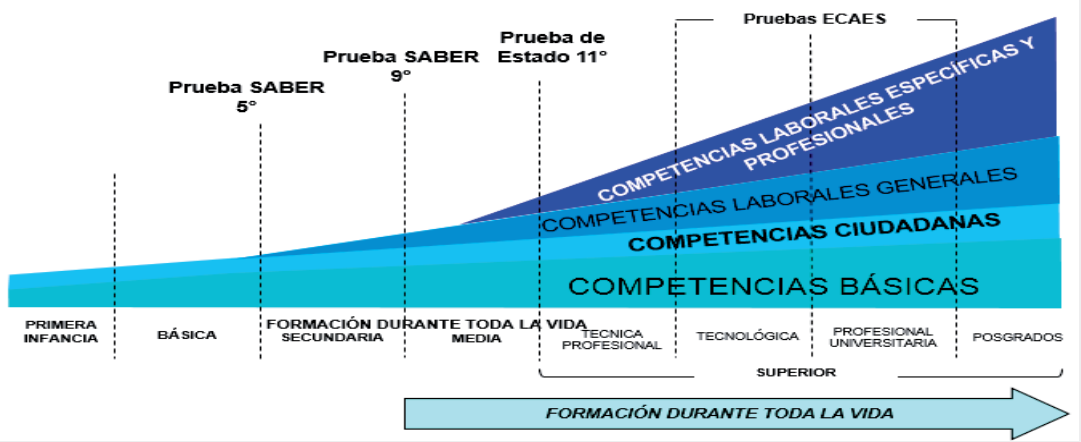

Figura 1. Política de calidad para la educación preescolar, básica y media 2007 - 2010 Fuente: [24]

Luego, el MEN [23] en los referentes y la evaluación de la calidad educativa, define una competencia como aquello que:

\footnotetext{
hace referencia a un conjunto de conocimientos, habilidades, actitudes, comprensiones y disposiciones cognitivas, metacognitivas, socioafectivas y psicomotoras apropiadamente relacionadas entre sí para facilitar el desempeño flexible, eficaz y con sentido de una actividad o de cierto tipo de tareas en contextos relativamente nuevos y retadores [p.10]
}

Se trae a colación que en relación a la competencia matemática, el MEN [24], pretendió con los estándares básicos en matemáticas que, unos cuantos procesos generales presentes en toda la actividad matemática, explicaran lo que se entiende por "ser matemáticamente competente", mostrando la variedad y riqueza de esta conceptualización para la organización de currículos focalizados en el perfeccionamiento de las habilidades matemáticas, ybuscando que éstas involucren los cinco procesos generales delaactividad matemática (formulary resolver problemas; modelar procesos y fenómenos de la realidad; comunicar; razonar, y formular comparar y ejercitar procedimientos y algoritmos). Dichos procesos generales están muy conexos con las competencias en su sentido más extenso, y también en el sentido limitado de "saber hacer en contexto", pues "ser matemáticamente competente" exige ser hábil, efectivo y eficiente en el desarrollo de cada uno de esos procesos generales, en los cuales cada estudiante va pasando por distintos niveles de competencia [24].

Además de involucrarse con esos cinco procesos, "ser matemáticamente competente" se materializa de manera concreta en el pensamiento lógico y el pensamiento matemático, el cual se subdivide en los cinco tipos de pensamiento propuestos en los Lineamientos Curriculares: el numérico, el espacial, el métrico o de medida, el aleatorio o probabilístico y el variacional. Estos cinco tipos de pensamiento mencionados antes, ofrecen elementos conceptuales generales que apoyan el diseño de situaciones de aprendizaje (y específicamente de situaciones problema) que involucren los diferentes pensamientos y que, a la vez, permitan que los procesos de aprendizaje de las matemáticas se generen a partir de la construcción de formas generales y articuladas de esos mismos tipos de pensamiento matemático [24]. 
No. 1
En consecuencia, el MEN con lo anterior propone un derrotero al docente de matemática para que al aplicar un currículo con lineamientos, estándares, indicadores, matrices, derechos básicos de aprendizajes, estrategias y metodologías de enseñanza y evaluaciones auténticas-formativas con resultados de aprendizaje en situaciones contextualizadas y vivenciales, sean correctamente funcionales para el aprendizaje del alumnado; e invita a que el mismo docente desarrolle y utilice su propia competencia, que no sólo es en Matemática, sino que también es una competencia matemática, muy diferente de la competencia de un matemático profesional o de un ingeniero [25] citando a [26],[27], [22].

Un ejemplo claro de que el sistema educativo colombiano está creando cada vez más y mejores espacios de aprendizaje para los estudiantes, es la tasa de mejora de la prueba $\mathrm{Pisa}^{4}$, ya que en el Resumen Ejecutivo Colombia 2015, El MEN-ICFES [28], reportan que nuestro país, obtuvo una de las más altas entre los países participantes en este ciclo, y mejorado notablemente su desempeño en las tres áreas evaluadas, Ciencias, Lectura y en Matemática, y ha avanzado 19 posiciones en comparación de resultados anteriores, lo cual indica al parecer que los niños y jóvenes están recibiendo educación de calidad y están aprovechando el tiempo en el aula. Y al hablar puntualmente de la competencia matemática ${ }^{5}$, el país creció 14 puntos, al avanzar de 376 en
2012 a 390 puntos en 2015. En consecuencia, Colombia aumentó una posición, superando a Perú y a Brasil en la región.

Sin embargo, a pesar de estos datos positivos, la OCDE alerta que el $66 \%$ de los estudiantes de Colombia no alcanzan los objetivos mínimos en matemáticas frente al $23 \%$ del resto de estados miembros que tampoco lo logra [28]. Las áreas relacionadas con matemáticas siguen siendo el punto en el que el país sale peor calificado. En relación a esto último, Ayala-García [29], concluyen que a pesar de que se lograron 390 puntos, solo por encima de República Dominicana, Perú y Brasil, aunque también por debajo de Chile, la Matemática es la evaluación en la que la población estudiantil colombiana presenta un pésimo desempeño entre las tres áreas que valora PISA. Y, es más, en contraste de lo que ocurre con el lenguaje, el resultado en Matemática tiene una pobre mejora con el paso de los años, y esto se sigue manifestando con los resultados publicados en el año 2015.

Al respecto [29], tomando datos del ICFES, se concluye que el $44 \%$ de los estudiantes colombianos termina la educación media sin desarrollar las tres competencias básicas en este conocimiento; mientras que PISA de acuerdo a su evaluación, revela que este indicador llega alarmantemente al $74 \%$ en el país. Tanto en Pisa como Saber se comprueban amplias y persistentes brechas de género que no tienen una explicación aceptable. Y es oportuno observar que cerca del $50 \%$ de las niñas no llega al nivel mínimo de la competencia matemática determinado en la prueba PISA, y que las brechas de género sean las más elevadas de todos los países participantes. Ante lo expuesto, y acorde a [29], se puede señalar que tanto las evaluaciones nacionales como las internacionales permiten determinar de manera preocupante la existencia de un persistente bajo nivel de competencia matemática en los estudiantes. 
En efecto, los repetitivos bajos resultados obtenidos en las pruebas estandarizadas sobre la competencia matemática en Colombia, muestran que se trata de uno de los conocimientos más incomprensibles que al estar relacionados con etiquetas académicas sin sentido práctico al mundo de la vida estudiantil, a ejemplo, las expresiones, leyes, reglas, fórmulas, figuras, estadísticas, teoremas de aprendizaje memorístico, ecuaciones, que inciden en hacer creer que es una especie de leviatán que infunde apatía o temor al estudiantado para desarrollarla.

La apreciación de D'Amore y Fandiño es tajante para el contexto educativo colombiano en relación a dicha competencia y a la evaluación de la Matemática, al decir que:

El mundo de la matemática escolar con frecuencia está lleno de estereotipos. Los estudiantes perciben la mayoría de las actividades (en cualquier nivel escolar) como una masa de mecanismos aparentemente inútiles que pareciera que no tienen ni pies ni cabeza. El por qué en los primeros años de la escuela superior, por ejemplo, los alumnos y los profesores (y por ende la sociedad) deben perder tiempo (y por lo tanto dinero público) en efectuar cálculos inútiles y repetitivos de expresiones inútilmente complejas, es para muchos un gran misterio [30].

Y aunque no hay estadísticas claras en el campo educativo colombiano en relación a las dificultades en la competencia matemática, Geary [31] establece que a nivel mundial se determina que del 5\% al $8 \%$ de los niños en edad escolar son identificados con problemas en esta competencia. Por suerte, muchos investigadores y educadores, al convertir la discapacidad y la competencia matemática en un tema de interés y pertinencia académica actual, están concentrando sus esfuerzos en comprender mejor las cuestiones que afrontan los estudiantes en su contexto escolar al presentar dificultades de aprendizaje en Matemática, a medida que se encuentran con los planes de estudios y pruebas internacionales-nacionales en los diferentes grados, que permiten una reflexión y búsqueda de respuestas a la experiencia escolar, aunque Abberley [32], advierte que a pesar de la trascendencia de dicho binomio, sigue evolucionando muy lentamente en la investigación educativa.

\subsubsection{Las discapacidades de aprendizaje y las DEAM}

Ahora bien, establecer un límite conceptual tanto para las DA como las DEAM es una tarea realmente difícil, polémica y compleja de precisar porque son rotuladas de diferentes maneras como dificultad de aprendizaje, problemas de aprendizaje, trastornos de aprendizaje, entre otros más, y por otro lado, a los amplios campos de investigación que están involucrados. Considera [33] que tradicionalmente la DA es vista: "desde una perspectiva académica, como el estudio de aquellos sujetos con determinadas características personales, que presentan problemas en el aprendizaje", de actividades y tareas escolares. Alude [33] que, en 1975, Kirk siendo presidente de la National Advisory Committee on Handicapped Children (NACHC), propone una definición de las DA que fue de gran impacto en el estudio de este ámbito:

\footnotetext{
Las dificultades de aprendizaje son un trastorno específico del desarrollo que significa un desorden en uno o más procesos psicológicos básicos implicados en la comprensión o el uso del lenguaje, escrito o hablado, que se manifiesta en una falta de habilidad para oír, pensar, hablar, leer, escribir, deletrear o en el cálculo matemático. El término incluye condiciones como hándicaps perceptivos, lesión cerebral, disfunción cerebral mínima, dislexia o afasia evolutiva. El término no incluye niños que tienen problemas de aprendizaje que son el resultado primario de dificultades de visión, audición o motrices, de retraso mental, de problemas emocionales o de desventajas ambientales, culturales o económicas [p.35]
}

Hammill [34] citado por [33], resume los elementos que han coincidido en las diversas definiciones de las DA que han marcado un camino y han ido variando desde 1960 hasta 1990, resultando más polémicas o más aceptadas para este ámbito de estudio (tabla I).
Enero-Diciembre 2016 ISSN 1794-8231 E-ISSN 2462 - 8794 PP: $121-140$

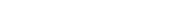


No. 1

Enero-Diciembre 2016 ISSN 1794-8231 E-ISSN 2462 - 8794

Tabla I. Definiciones de las DA

\begin{tabular}{|c|c|c|c|c|c|c|c|c|c|}
\hline Definicion & $\mathbf{1}$ & $\mathbf{2}$ & $\mathbf{3}$ & $\mathbf{4}$ & $\mathbf{5}$ & $\mathbf{6}$ & $\mathbf{7}$ & $\mathbf{8}$ & $\mathbf{9}$ \\
\hline Kirk (1962) & Intra-Individual & $\mathrm{Si}$ & $\mathrm{Si}$ & $\mathrm{Si}$ & $\mathrm{Si}$ & $\mathrm{Si}$ & $\mathrm{No}$ & $\mathrm{NO}$ & $\mathrm{Si}$ \\
\hline Bateman(1965) & Aptitud-Ejecucion & $\mathrm{No}$ & $\mathrm{Si}$ & $\mathrm{No}$ & $\mathrm{No}$ & $\mathrm{No}$ & $\mathrm{No}$ & $\mathrm{NO}$ & $\mathrm{Si}$ \\
\hline NACHC(1975) & Intra-Individual & $\mathrm{Si}$ & $\mathrm{Si}$ & $\mathrm{No}$ & $\mathrm{Si}$ & $\mathrm{Si}$ & $\mathrm{Si}$ & $\mathrm{NO}$ & $\mathrm{Si}$ \\
\hline ACLD(1986) & Intra-Individual & $\mathrm{Si}$ & $\mathrm{No}$ & $\mathrm{Si}$ & $\mathrm{Si}$ & $\mathrm{Si}$ & $\mathrm{Si}$ & $\mathrm{Motriz}$ & -- \\
\hline ICLD(1987) & Intra-Individual & $\mathrm{Si}$ & $\mathrm{No}$ & $\mathrm{Si}$ & $\mathrm{Si}$ & $\mathrm{Si}$ & $\mathrm{Si}$ & HH. Sociales & $\mathrm{Si}$ \\
\hline NJCLD(1988) & Intra-Individual & $\mathrm{Si}$ & $\mathrm{No}$ & $\mathrm{Si}$ & $\mathrm{Si}$ & $\mathrm{Si}$ & $\mathrm{Si}$ & $\mathrm{No}$ & $\mathrm{Si}$ \\
\hline
\end{tabular}

NOTA:1=Causa, 2=Disfunción Cerebral Mínima, 3=Procesos, 4=Toda la Vida, 5=Lenguaje, 6=Académico, $7=$ Pensamiento, $8=$ Otros, $9=$ Consideración multihándicaps

Fuente: Revisión sobre los elementos considerados en las definiciones de las DA (tomado de Hammill, 1990 y de González, p. 38, 2012)

Una interesante conceptualización operativa de las DA que trata de lograr un cierto consenso en determinadas cuestiones discutibles, proponen Fiuza y Fernández [35], expresando que son:

[...] aquellas dificultades de aprendizaje que están constituidas por un conjunto heterogéneo de problemas cuyo origen es, probablemente, una disfunción del sistema nervioso central. Se manifiestan primariamente con problemas en el ámbito lingüístico y con defectos de procesamiento en los principales factores cognitivos (atención, percepción, memoria), derivadamente, en el ámbito de las disciplinas instrumentales básicas (lectura, escritura, matemáticas) y, secundariamente, en las diversas áreas curriculares (ciencias experimentales, ciencias sociales, segundo idioma). Cursan, además, con problemas de personalidad, autoconcepto y sociabilidad, y pueden ocurrir a lo largo del ciclo vital del sujeto, de acuerdo a Santiuste y González [36], citado por [35, p.25].
Esta definición es valiosa según [35], porque: a). contempla los factores neurológicos, biológicos y genéticos; b). Destaca que las DA se manifiestan en dificultades lingüísticas de comprensión y producción referidos a todos los componentes del lenguaje; c). Se hace una doble referencia curricular, primero a las disciplinas instrumentales y después al resto de áreas curriculares; y se destaca para estos autores que las DA no son efecto, sino causa de problemas de personalidad, sociales y/o culturales. González [33] citado por [35], resume las características de un sujeto con DA en la tabla II.

Tabla II. Resumen las características de un sujeto con DA

\begin{tabular}{|l|}
\hline Grupo Heterogeneo \\
\hline Dificultades de adaptacion al sistema educativo \\
\hline Dificultades para adquirir nuevos conocimientos, destrezas o estrategias \\
\hline Dificultades en la resolucion de problemas o tareas acadeicas \\
\hline Deficiencias en estrategias de aprendizaje o tareas academicas \\
\hline Deficiencias en estrategias de aprendizaje (uso, consciencia y velocidad) \\
\hline Retraso leve en el desarrollo \\
\hline Bajo rendimiento en lenguaje, lectura, escritura y matematicas \\
\hline $\begin{array}{l}\text { Presumibles problemas en procesos Psicologicos: atencion, memoria, percepcion, linguisticos, razonamiento, cognicion social (autoestima, } \\
\text { auto concepto, interaccion social, motivacion, expectativas de éxito para la resolucion de problemas) y metacognicion }\end{array}$ \\
\hline Presumiblemente discrepancia CI-rendimiento/discrepancia edad-rendimiento/discrepancia comprencion verbal-rendimiento \\
\hline Las dificultades pueden aparecer a lo largo del ciclo vital \\
\hline Inteligencia normal \\
\hline Problemas de inteligencianpractica \\
\hline No responde al tratamiento temprano \\
\hline Dificultrades no causadas por retraso mental, deficit sensoriales y matrices, desajustes socioemocional o deficiencias socioculturales \\
\hline Presumible existencia de disfunciones neurologicas \\
\hline
\end{tabular}

En cuanto a las clasificaciones existentes sobre DA, la más ampliamente aceptada, actualizada y utilizada por los investigadores es la del Manual Diagnóstico y Estadístico de los Trastornos Mentales de la quinta edición (DSM-5 TM; APA, 2013) [37]. El DSM-5 TM que al compararse con el DSM-IV TR, APA, 2002 [38], cambia la denominación de trastorno del aprendizaje (antes trastornos de las habilidades académicas) por la de trastorno específico del aprendizaje (specific learning disorder), aunque los trastornos son similares, pero ahora son agrupados en un solo diagnóstico, tal y como muestra la tabla III. 
Tabla III. Tabla comparativa del Manual Diagnóstico y Estadístico de los Trastornos de Retardo o Trastornos Mentales del aprendizaje (DSM-TR/TM) de la Asociación Americana de Psiquiatría (APA) en sus versiones IV y 5

\begin{tabular}{|l|l|}
\hline \multicolumn{1}{|c|}{ DSM-IV-TR (APA, 2002) } & \multicolumn{1}{c|}{ DSM-5-TM (APA, 2013) } \\
\hline Trastorno del Aprendizaje & \multicolumn{1}{c|}{ Trastorno Especifico del Aprendizaje } \\
\hline Trastorno de lectura & $\begin{array}{l}\text { Trastorno especifico de aprendizaje con: } \\
\text {-deterioro de la lectura } \\
\text {-deterioro de la expresión escrita } \\
\text {-deterioro de la matemática }\end{array}$ \\
\hline Trastorno de la expresión escrita & \\
\hline Trastorno del calculo & $\begin{array}{l}\text { Se debe especificar la gravedad del trastorno en: leve, moderado, } \\
\text { grave }\end{array}$ \\
\hline & $\begin{array}{l}\text { Se han de cumplir los 4 criterios diagnósticos y con un mínimo de seis } \\
\text { meses para determinar un trastorno especifico de aprendizaje } \\
\text { basándose en una síntesis clínica de la historia del individuo (del } \\
\text { desarrollo, médica, familiar, educativa), informes escolares y } \\
\text { evaluación psicoeducativa. }\end{array}$ \\
\hline & $\begin{array}{l}\text { Procedimiento de registro. Se debe registrar cada una de las áreas } \\
\text { académicas y subaptitudes afectadas del trastorno. }\end{array}$ \\
\hline
\end{tabular}

Fuente: Autor

En relación a las alteraciones en el cálculo, el DSM-5 TM sostiene las alteraciones en el cálculo además de las alteraciones en conceptos matemáticos básicos, incorporando: las dificultades en solucionar problemas matemáticos; extiende los criterios para la evaluación; se puede emplear discalculia como concepto opcional, pero se debe detallar cualquier dificultad adicional presente, como dificultad de comprensión de la lectura o del razonamiento matemático;

Tabla IV. Diagnóstico de los trastornos específicos del aprendizaje escolar

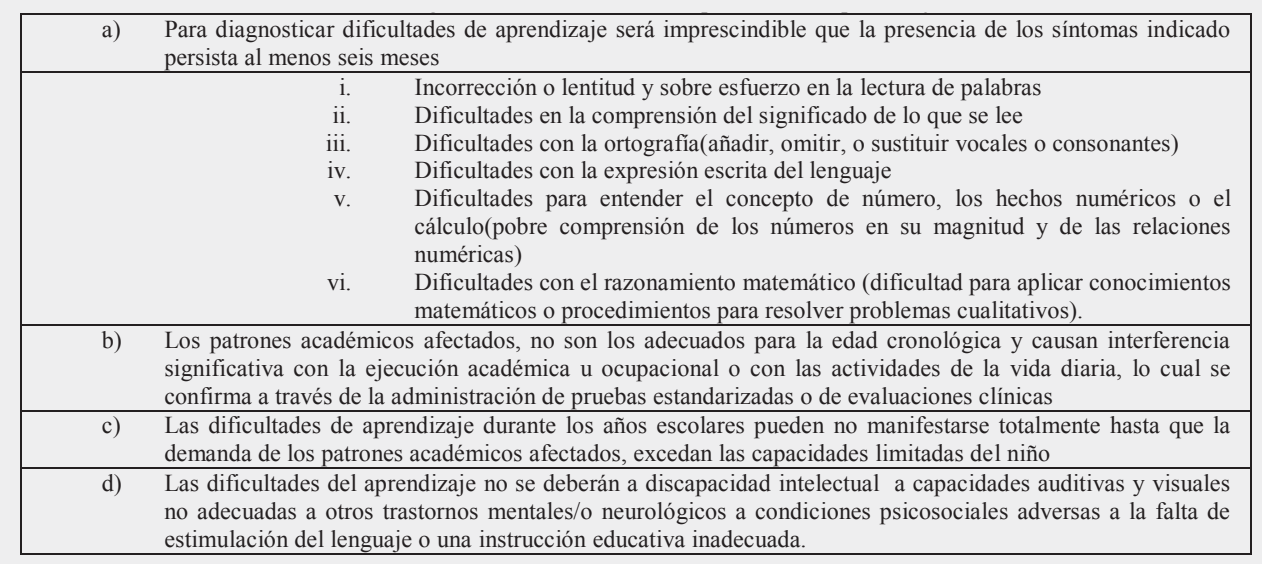

Fuente: Criterios diagnósticos y de exclusión de los trastornos del aprendizaje escolar según el DSM-V TM (APA, 2013)

\subsubsection{Concepto, causas, características, evaluación e intervención de las DEAM}

Y al acercarnos al terreno de las DEAM, se advierte que los estudios para entender lo que significa una dificultad en Matemática o qué se puede hacer en este ámbito, están rezagadas en relación con las y por ultimo aparece el procedimiento de registro, como herramienta que permite y obliga a registrar cada una de las áreas académicas y subaptitudes afectadas del trastorno. En la tabla IV, se presenta una síntesis de cómo habría que diagnosticar a los trastornos específicos del aprendizaje escolar de acuerdo al DSM-5 TM, concordando en parte con algunas cuestiones planteadas en las definiciones anteriores. investigaciones hechas en el campo de la lectura. Y lo anterior ya lo confirmaba, [39], que manifestaba en relación a la DEAM lo siguiente:

ISSN 1794-8231

-ISSN $2462-8794$

PP: $121-140$
[...] la explicación de las actitudes negativas y bajos rendimientos en matemáticas es mucho más complicada y menos unánime. ¿Son objetivamente difíciles las matemáticas o más bien sucede que no se enseñan bien?, ¿qué origen y significado tienen las enormes diferencias en la competencia 
No. 1 matemática de los alumnos?, ¿hay alumnos que sufren alguna clase de alteración o trastorno real-por ejemplo, la clásica "discalculia" qué les impide o dificulta el aprendizaje de las operaciones matemáticas más elementales?, ¿por qué son tan difíciles las matemáticas para tantos alumnos que no llegan a ese grado de supuesta alteración?...y, sobre todo, ¿qué hacer con esta situación?, ¿cómo puede el profesor enfrentarse a ella? Los conocimientos actuales sobre dificultades en el aprendizaje de las matemáticas sólo dan respuestas parciales e incompletas a estas preguntas. [ p.2]

\subsubsection{Concepto de DEAM}

Tal como lo señalan [35], se considera a las DEAM como: "dificultades en el aprendizaje de la matemática que conllevan el análisis de la problemática asociada a factores cognitivos y/o educativos de los que puedan derivarse dificultades en el manejo de los conceptos y operaciones numéricas y lógicomatemáticas".
Siguiendo [40], las DEAM son englobadas como los trastornos de cálculo y los trastornos en la resolución de problemas, $\mathrm{y}$ son diagnosticadas cuando: a). El nivel de rendimiento académico en matemáticas del sujeto, aun teniendo un CI medio (entre 75 y 120) y una escolaridad correcta, se sitúa por debajo de lo esperado por su edad cronológica y por su nivel de desarrollo mental. b). Cuando el bajo rendimiento académico no puede ser atribuido a un déficit sensorial (motórico, visual y/o auditivo). La tabla $\mathrm{V}$ presenta, dentro del trastorno específico del aprendizaje, las dificultades en las matemáticas siguiendo el DSM-V TM.

Tabla V dificultades en las matemáticas siguiendo el DSM-V TM.

\begin{tabular}{|l|}
\hline Dificultades en el concepto de numero \\
\hline Difícultades en la memorización de los hechos aritméticos \\
\hline Dificultades en la corrección y fluencia en el calculo \\
\hline Dificultades en el razonamiento matemático \\
\hline NOTA: Discalculia es un término alternativo usado para referirse a un patrón de dificultades caracterizado por problemas en el procesamiento de la \\
información numérica, el aprendizaje de acciones aritméticas y la ejecución correcta y fluida del cálculo matemático. Si la discalculia es usada para \\
especificar este particular patrón de dificultades matemáticas, es importante especificar también algunas otras dificultades que se suelen presentar \\
asociadas a este problema, como son las dificultades en el razonamiento matemático o en el reconocimiento y razonamiento de las palabras, por ejemplo \\
en el enunciado de un problema.
\end{tabular}

Fuente: Subhalidades del trastorno especifico del aprendizaje que puedan estar alteradas en las matemáticas Según el DSM V TM (APA, 2013) citado por [35]

Para el DSM-V TM, la discalculia es un concepto que se ha usado por muchos años para referirse a una DEAM. Discalculia se puede comprender de manera general como un déficit grave o total para calcular. Y aunque un estudiante con discalculia puede memorizar y recitar números, muchas veces puede que no esté desarrollando el "sentido numérico" que es esencial para el aprendizaje futuro de las matemáticas. Algunos teóricos emplean el concepto discalculia para explicar a niños con problemas para aprender conceptos y técnicas matemáticas. Sin embargo, en la actualidad es más común usar el término DEAM.

\subsubsection{Causas de las DEAM}

En cuanto a las explicaciones del origen de las DEAM, cada enfoque teórico postula diferentes causas, Kelly en Understood. org $^{6}$, opina que las DEAM suelen pasar frecuentemente desapercibidas en la infancia porque los niños están aprendiendo habilidades básicas a través de la memorización, lo que complica su identificación temprana. Para [40], citado por [35], señalan cuatro enfoques: evolutivo, educativo, neurológico y cognitivo; de ellos, los dos primeros consideran que las causas son externas al sujeto, mientras que los dos segundos plantean que son internas: a). Evolutivo: se defiende la importancia de la estimulación que recibe el niño en las primeras etapas del desarrollo en la aparición o no de las DEAM; b). Educativo: se pone el énfasis en la dificultad propia de la asignatura

${ }^{6} \mathrm{~K}$. Kelly, "Cómo las diferentes dificultades de aprendizaje y de atención pueden causar problemas con las matemáticas", [Documento en línea en un blog]. Disponible en https://www.understood.org/es$\mathrm{mx} /$ learning-attention-issues/child-learning-disabilities/dyscalculia/ difference-between-dyscalculia-math-troubles-associated-withdyslexia-dyspraxia. [Accedido: 10-nov-2015] 
y de su enseñanza, en la forma de intervenir para dar respuesta a la diversidad de aptitudes, actitudes e interés del alumno; c). Neurológico: asocia lesiones en determinadas áreas cerebrales con las DEAM, asumiendo que son trastornos adquiridos como consecuencia de una lesión cerebral sufrida después de que las habilidades matemáticas hayan sido dominadas; $\mathrm{y}$, por último, d). Cognitivo: las DEAM son producidas por procesos cognitivos inadecuados. De lo anterior, se puede establecer estudiantes con DEAM que emplean de forma equivocada sus recursos atencionales, que no desarrollan de forma correcta los procesos de recuerdo, almacenamiento, etc., o niños que no tienen los conocimientos previos indispensables para realizar la tarea requerida. Todo esto se vincula con un procesamiento de información desfasado de los requerimientos mentales.

Es imprescindible mencionar a un investigador que tiene importantes repercusiones en la práctica educativa como Padget [41] citado por [33], quien identifica las características principales de cada uno de los tipos de DA y las consecuencias o síntomas secundarios asociados, que pueden establecer diagnósticos diferenciales, al clasificar, por ejemplo, a las DEAM, señalando que se revelan en sujetos con CI verbal medio, con una media en comprensión auditiva, expresión oral, identificación de palabras y comprensión lectora. Los síntomas primarios para [41] son las dificultades en el cálculo, en comprensión y resolución de problemas aritméticos y en la escritura legible, que subyacen a los problemas viso-espaciales y de razonamiento. Los síntomas secundarios hacen referencia a [41], a los de expresión escrita. Las áreas que pueden estar afectadas en el trastorno del cálculo son las siguientes: lingüísticas, perceptivas, atencionales y las propiamente matemáticas.

Los niños con DEAM según [41], en la educación primaria se producen al presentarse dificultades que guardan relación con la atención, la percepción, el procesamiento auditivo, la memoria, el autoconcepto, las atribuciones, la conducta, estados de ansiedad $\mathrm{y}$ estrategias metacognitivas.

\subsubsection{La evaluación de las DEAM}

En la evaluación formal de las DEAM, informan [35], se utilizan pruebas psicológicas (para medir los procesos cognitivos y neuropsicológicos que intervienen en la realización de tareas matemáticas) y pruebas psicopedagógicas (que no se diferencian esencialmente de las que puede ejecutar cualquier profesor pero están estandarizadas para comparar resultados con los baremos de la población de la misma edad), entre las que se pueden citar las de [36] y otras propias del aula misma como evaluaciones auténticas, test de pruebas estandarizadas en el aula de las habilidades académicas, actividades del proceso enseñanza-aprendizaje y con el análisis de los resultados de aprendizajes. Y en cuanto a las pruebas psicológicas, se puede entre muchas, la más utilizada por la mayoría de profesionales, las escalas de inteligencia como el WISC-IV, de Weschler [42] citado por [35], para niños y adolescentes de 6 a 16 años, que incluyen una prueba de aritmética y otra de memoria auditiva inmediata (dígitos).

\subsubsection{Pautas de intervención psicoeducativa en el aula}

Las pautas a seguir en el diseño de programas de enseñanza-aprendizaje para los alumnos con DEAM para [35], se agrupan principalmente en la prevención y desarrollan una metodología basada en la teoría cognitiva y un modelo de respuesta e intervención temprana, al considerar que éste es el marco más sólido para sustentar las decisiones que un docente puede tomar de un modo permanente durante su labor de enseñanza. Se pueden tomar dos orientaciones o pautas de intervención según [35], acorde a la necesidad de adaptar el proceso de
Enero-Diciembre 2016 ISSN 1794-8231 E-ISSN 2462 - 8794 PP: $121-140$ 
enseñanza-aprendizaje a las características de los alumnos, lo que supone individualizar el proceso educativo en dos aspectos: primero, un análisis de las diferentes tareas y procesos matemáticos que se pretende enseñar con el fin de averiguar cuáles son los prerrequisitos que el alumno debe dominar antes de comenzar el aprendizaje de dicha tarea. Y segundo, hacer que el alumno utilice todos los sentidos en el aprendizaje de una tarea; a partir de la manipulación de objetos de la vida cotidiana antes de comenzar con la utilización de símbolos.

\section{Conclusiones}

Se reconoce que un grupo de estudiantes muestra problemas para aprender técnicas, estrategias, métodos y conceptos de la competencia matemática que persisten a través de los años escolares y de la adultez. Está claro que las dificultades concretas en las áreas de memoria, desarrollo cognoscitivo, y capacidad visual-espacial favorecen la aparición de las DEAM. En general, una $\mathrm{PcD}$ en Matemáticas tiene problemas para avanzar académicamente en la escuela al mismo ritmo que sus compañeros, a pesar de que se hayan efectuado prácticas de enseñanza efectivas a través del tiempo. No obstante, las investigaciones en curso sobre los problemas en Matemática, no logran determinar aun hoy cómo la DEAM incide en la capacidad de una persona para aprender Matemática en las diferentes áreas. Hoy en día, la mayoría de las investigaciones se han focalizado sobre todo en las técnicas asociadas con los cálculos matemáticos como los números, contar y la aritmética y en la resolución de problemas de lógica. También se conoce poco sobre el desarrollo y las dificultades en áreas como álgebra, geometría, mediciones, y análisis de datos y probabilidades.

Es preciso señalar que la concepción de las DA en Colombia sufre de formulaciones ambiguas y de falta de acuerdo acerca de qué sean, cuál es la causa que las origina, qué personas las padecen, que consecuencia se presentan, cuales son los criterios diagnósticos, cuales son pautas de evaluación e intervenciones unificadas y estables que faciliten la tarea de los profesionales de la educación. Lamentablemente hay muy pocos programas de prevención de las DEAM, con escasos manuales de intervención y no hay claridad en los cuadros clasificatorio dentro de las NEE que sirviera de marco de referencia a los profesionales, a los organismos de la salud, de la educación y de la administración pública para poder atender con similares criterios y recursos las necesidades de los alumnos que sufren las DEAM. Observando Gonzales [43], la necesidad de una educación inclusiva con respuestas formativas integrales y eficaces que utiliza "diferentes alternativas de acceso al conocimiento y evalúa diferentes niveles de competencia", en esta condición discapacitante.

Para diversos autores apoyados en el modelo educativo psicopedagógico de las DA, consideran que mediante un diagnóstico, seguimiento y evaluación educativa permanente con criterios que orienten la corrección de las dificultades basados en aprendizajes que desarrollen la creatividad, las competencias, la organización y adaptación de contenidos en Matemática junto a la evaluación autentica y los resultados de aprendizaje como herramientas pedagógicas, entre otras, permiten establecer a los docentes las medidas curriculares y organizativas necesarias para atender las NEE que presenta el alumnado con DEAM. Esto ofrece la posibilidad de desarrollar una propuesta curricular específica y personalizada sin mayores sofisticaciones paraunaidentificación temprana, prevención e intervención oportuna en los primeros grados escolares. Luego es imprescindible la aprobación de una ley que establezca el modo de ayudar a los estudiantes a superar las dificultades que encuentran en su aprendizaje marcando un hito histórico en el 
desarrollo del campo de las DA en el país, y que las reconozcan especialmente como una categoría diagnóstica específica dentro del alumnado con NEE. Con esto, se podría en la educación primaria desarrollar con mayor énfasis la atención individualizada para la prevención por ejemplo, de las DEAM, y en la puesta en práctica de mecanismos de refuerzo tan pronto como se detecten estas dificultades en los primeros grados.

En definitiva, es fundamental el reconocimiento, concreción y desarrollo de la categoría de DA y de las DEAM a nivel legislativo $\mathrm{y}$ en las políticas públicas en Colombia, para ser llevadas a las administraciones educativas $y$ otras entidades, que supere realmente el modelo individual o médico en la cual la discapacidad es un problema de la persona, a un modelo biopsicosocial en la que el nivel de discapacidad esta dado en la concepción de lo que una persona experimenta o en otras palabras, la función entre la persona y el medio ambiente. Esto se daría mediante una sólida fundamentación teórico y empírico proporcionada a nivel científico, y con activa participación por parte de los educadores como investigadores en el aula en el estudio de las discapacidades, en la que se tenga en cuenta que una DEAM se da en un contexto determinado en el cual es necesario el respeto al tiempo y a los ritmos de aprendizaje para una $\mathrm{PcD}$ que necesita alcanzar unos resultados de aprendizaje satisfactorios en un sistema educativo integral e incluyente que permita aprendizajes acordes a sus capacidades, intereses e intervenciones psicoeducativas adecuadas.

\section{Referencias}

[1] D. Geary, C. Hamson, and M. Hoard, "Numerical and arithmetical cognition: a longitudinal study of process and concept deficits in children with learning disability", Journal of Experimental
Child Psychology, vol. 77, pp.236-263, 2000

[2] M. Blanco, Dificultades Específicas del Aprendizaje de las Matemáticas en los primeros años de la escolaridad: detección precoz y características evolutivas, Ministerio de Educación, España: Colección Investigación, 2009.

[3] L. Siegel, "Issues in the definition and diagnosis of learning disabilities: a perspective on Guckenberger versus Boston University", Journal of Learning Disabilities, vol. 32 no. 4, pp.304-19, 1999

[4] Universidad Pedagógica Experimental Libertador, "Vicerrectorado de Investigación y Postgrado", Manual de Trabajos de Grado de Especialización, Maestrías y Tesis Doctorales. FEDUPEL Caracas: 2014.

[5] J. Muñoz, I. Novo y E. Espiñeira, (2013, Jun. 24) Estudios sobre educación "La inclusión de los estudiantes universitarios con discapacidad en las universidades presenciales: actitudes e intención de apoyo por parte de sus compañeros", en, [En línea] Disponible en http://dadun. unav.edu/bitstream/10171/29566/2/ MUN\%C3\%8C\%C6\%92OZ\%20_\%20 NOVO.pdf.

[6] Organización Mundial de la Salud. Discapacidad y salud. Nota descriptiva [En línea]. Disponible en http://www. who.int/mediacentre/factsheets/fs352/ es/. [Accedido: abril 3 de 2015].

[7] Departamento Administrativo Nacional de Estadística (DANE). (2012). Encuesta Nacional de Calidad de Vida. [En línea]. Disponible en http://www.dane.gov.co/index.php/ estadisticassociales/calidad-de-vidaecv.
Enero-Diciembre 2016 ISSN 1794-8231 E-ISSN 2462 - 8794 PP: $121-140$ 
[9] K. Schriner, "A disability studies perspective on employment issues and policies for disables people. An internacional view", en G. L. Albrecht, K. D. Seelman y M. Bury, Handbook of Disability Studies. Sage Publications, University of Illinois at Chicago 2001.

[10] C. Gómez Acosta, Conceptualización de discapacidad: reflexiones para Colombia, Bogotá: Universidad Nacional de Colombia. Facultad de Medicina, 2007

[11] Naciones Unidas Derechos Humanos. (2014). Convención sobre los Derechos de las Personas con Discapacidad. Guía de formación. Serie de capacitación profesional $N^{o} 19$, Nueva York y Ginebra: Naciones Unidas.

[12] E. Brandt and A. Pope, Enabling America: assessing the role of rehabilitation science and engineering. Washington: National Academy Press, 1997

[13] S. Mitra, "The capability approach and disability", Journal of Disability Police Studies, vol. 16, No. 4, pp. 236-247, 2006.

[14] Organización Mundial de la Salud. (2001). CIF, Clasificación Internacional del Funcionamiento, de la Discapacidad y de la Salud. España: Ministerio de Trabajo $y$ Asuntos Sociales. Secretaría de Estado de Servicios Sociales, Familias y Discapacidad. Instituto de Mayores y Servicios Sociales (IMSERSO)

[15] C. Cuervo y A. Trujillo, "Avances conceptuales e investigativos de la maestría en Discapacidad e Inclusión
Social en la Universidad Nacional de Colombia, en Cuervo", C., Trujillo, A., Vargas, D., Ángel, L., Mena, B. ed. 2004. Discapacidad e inclusión social: reflexiones desde la Universidad Nacional de Colombia. Bogotá: UNAL de Colombia, 2005.

[16] M. Moreno, Concepciones y políticas en discapacidad: Un binomio por explorar. Bogotá D.C: Facultad de Medicina, Universidad Nacional de Colombia, 2007

[17] L. Garay, Colombia: entre la exclusión y el desarrollo. Propuestas para la transición al Estado Social de Derecho, Bogotá: Contraloría General de la República, 2002

[18] Ministerio de Educación Nacional. (2004). Educación para cada situación. Bogotá: Altablero No. 28, marzoabril, 2004 [Online]. Available: http:// www.mineducacion.gov.co/1621/ article-87346.html.

[19] A. Martínez, A. Uribe y H. Velázquez, "La discapacidad y su estado actual en la legislación colombiana", Duazary, vol. 12, no 1, pp. 49 -58, 2015, junio.

[20] C. Gómez y C. Cuervo, "Conceptualización de discapacidad: reflexiones para Colombia", tesis de Maestría en Discapacidad e Inclusión Social, Bogotá: Universidad Nacional de Colombia, Facultad de Medicina, 2007.

[21] C. Cordeu, "Reflexiones en torno a la inclusión sociocomunitaria de personas con discapacidad intelectual: escuchando la voz de sus protagonistas" tesis de Maestría en Psicología, Universidad de Chile, Facultad de Ciencias Sociales, Santiago de Chile, 2008. 
[22] B. D’Amore, J. Díaz Godino y M. Fandiño. Competencias y matemática, Bogotá: Cooperativa Editorial Magisterio, 2008.

[23] MEN. (2006), Referentes y Evaluación de la Calidad Educativa, Ministerio de Educación Nacional [En línea]. Disponible en http://www. mineducacion.gov.co/cvn/1665/ articles-246512_archivo_pdf_ MauricioMartinez.pdf,

[24] MEN. (2006) Estándares básicos de competencias en matemáticas, Imprenta Nacional de Colombia [En línea]. Disponible en http:// www.mineducacion.gov.co/1621/ articles-116042_archivo_pdf2.pdf.

[25] B. D’Amore y M. Fandiño, "Propuestas metodológicas que constituyeron ilusiones en el proceso de enseñanza de la matemática", Educación Matemática, vol.27, pp. 7-43, 2015.

[26] M. Fandiño, “'Diventare competente', una sfida con radici antropologiche", La matematica e la sua didattica, vol. 17, no3, pp. 260-280, 2003

[27] M. Fandiño, Currículo, evaluación y formación docente en matemática, Bogotá: Cooperativa Editorial Magisterio, 2006

[28] MEN-ICFES. (2015). Resumen Ejecutivo Colombia en PISA 2015, Bogotá D.C. [En línea]. Disponible en http://www.icfes.gov.co/docman/ institucional/home/2785-informeresumen-ejecutivo-colombia-enpisa-2015

[29] J. Ayala-García. (2015). Documentos de Trabajo Sobre Economía Regional, Cartagena: Banco de la República. [En línea]. Disponible en http:// www.banrep.gov.co/docum/Lectura finanzas/pdf/dtser_217.pdf

[30] B. D’Amore y M. Fandiño. (2015) "La Matemática en las Aulas de Primera Infancia", XVIII Congreso Internacional de Educación Inicial, Neiva: Confederación Nacional por la Infancia de Colombia.

[31] D. Geary, "Mathematics and Learning Disabilities", Journal of Learning Disabilities, vol. 37, pp. 4-15, 2004

[32] P. Abberley, "The Concept of Oppression and the Development of a Social Theory of Disability", Disability, Handicap and Society, vol 2, no 1, pp.519, 1987.

[33] M. González, (coord.), Prevención de las dificultades de aprendizaje, Madrid: Pirámide, 2012

[34] D. Hammill, On defining learning disabilities: an emerging consensus, Journal of learning disabilities, vol. 23, no 2, pp. 74-84, 1990

[35] M. Fiuza y M. Fernández, (2014) Dificultades de aprendizaje y trastornos del desarrollo. Manual didáctico, Madrid: Ediciones Pirámide

[36] V. Santiuste y J. González-Pérez, Dificultades de aprendizaje $e$ intervención psicopedagógica. Madrid: Editorial CCS, 2005

[37] Asociación Americana de Psiquiatría, (APA), Manual Diagnóstico y Estadístico de los Trastornos Mentales, DSM-V-TM $5^{\mathrm{a}}$ ed., Washington, DC: Panamericana, 2013.

[38] Asociación Americana de Psiquiatría, (APA), Manual Diagnóstico y Estadístico de los Trastornos Mentales, DSM-IV-TR (4 ${ }^{a}$ ed.), Washington, DC: Panamericana, 2002.
Enero-Diciembre 2016 ISSN 1794-8231

E-ISSN 2462 - 8794 PP: $121-140$ 
No. 1

Enero-Diciembre 2016

ISSN 1794-8231

E-ISSN 2462 - 8794 PP: $121-140$

[39] A. Rivière, "Problemas y dificultades en el aprendizaje de las matemáticas: una perspectiva cognitiva", in Desarrollo psicológico y educación, III. Necesidades educativas especiales $y$ aprendizaje escolar, A. Marchesi, C. Coll and J. Palacios Alianza: Madrid, 1990.

[40] A. Pérez, P. Poveda y M. López, "Dificultades de aprendizaje y trastornos del cálculo", en J. L. Castejón y L. Navas, Dificultades y trastornos del aprendizaje y del desarrollo en infantil y primaria, Alicante: ECU, 2011

[41] S. Padget, "Lessons from research on Dislexia: Implications for a classification system for learning disabilities", Learning Disability Quartely, vol. 21, pp.167-178, 1998

[42] D. Wechsler, WISC-IV. Escala de inteligencia de Wechsler para niños- $I V$, $4^{\mathrm{a}}$ ed., Madrid: Pearson, 2012.

[43] R.M. González. Motivación para la "inclusión", características y necesidades de los estudiantes y docentes en la universidad. (Aplicativo con análisis de gráficos estilísticos). Revista Ecomatemático, vol. 6, no. 1, pp. 107-115, 2015. 